\title{
Determination of the solid-liquid interfacial free energy along a coexistence line by Gibbs-Cahn integration
}

\author{
Brian B. Laird, ${ }^{1, a)}$ Ruslan L. Davidchack, ${ }^{2}$ Yang Yang, ${ }^{3,4}$ and Mark Asta ${ }^{4}$ \\ ${ }^{1}$ Department of Chemistry, University of Kansas, Lawrence, Kansas 66045, USA \\ ${ }^{2}$ Department of Mathematics, University of Leicester, Leicester LE1 7RH, United Kingdom \\ ${ }^{3}$ Department of Physics, East China Normal University, Shanghai 200062, China \\ ${ }^{4}$ Department of Chemical Engineering and Materials Science, University of California, Davis, \\ California 95616, USA
}

(Received 15 July 2009; accepted 27 August 2009; published online 18 September 2009)

\begin{abstract}
We calculate the solid-liquid interfacial free energy $\gamma_{s l}$ for the Lennard-Jones (LJ) system at several points along the pressure-temperature coexistence curve using molecular-dynamics simulation and Gibbs-Cahn integration. This method uses the excess interfacial energy $(e)$ and stress $(\tau)$ along the coexistence curve to determine a differential equation for $\gamma_{s l}$ as a function of temperature. Given the values of $\gamma_{s l}$ for the (100), (110), and (111) LJ interfaces at the triple-point temperature $\left(T^{*}\right.$ $=k T / \epsilon=0.618)$, previously obtained using the cleaving method by Davidchack and Laird [J. Chem. Phys. 118, 7657 (2003)], this differential equation can be integrated to obtain $\gamma_{s l}$ for these interfaces at higher coexistence temperatures. Our values for $\gamma_{s l}$ calculated in this way at $T^{*}=1.0$ and 1.5 are in good agreement with those determined previously by cleaving, but were obtained with significantly less computational effort than required by either the cleaving method or the capillary fluctuation method of Hoyt, Asta, and Karma [Phys. Rev. Lett. 86, 5530 (2001)]. In addition, the orientational anisotropy in the excess interface energy, stress and entropy, calculated using the conventional Gibbs dividing surface, are seen to be significantly larger than the relatively small anisotropies in $\gamma_{s l}$ itself. (C) 2009 American Institute of Physics. [doi:10.1063/1.3231693]
\end{abstract}

As defined by Gibbs in his classic work on surface thermodynamics, ${ }^{1}$ the interfacial free energy $\gamma$ is the reversible work required to create a unit area of interface. For solid-liquid interfaces, this quantity plays a central role in understanding a number of technologically important phenomena in physics, chemistry, and materials science, such as crystal nucleation and growth, ${ }^{2-4}$ dendritic solidification, ${ }^{5,6}$ liquid-metal embrittlement, ${ }^{7}$ and wetting. ${ }^{8}$ Despite its importance, direct experimental measurements, which usually involve challenging contact angle studies, are relatively few in number ${ }^{9}$ and, with a few exceptions, ${ }^{10,11}$ are not precise enough to resolve the often small orientation dependence (anisotropy). Because of the lack of experimental data, much of our current understanding of the material dependence of the solid-liquid interfacial free energy $\gamma_{s l}$ comes via atomistic simulations. ${ }^{12}$

The determination of $\gamma_{s l}$ in a computer simulation is complicated by the fact that the solid can support nonhydrostatic stress ${ }^{13}$-this precludes the use of mechanical approaches commonly used for liquid-vapor or fluid-wall interfaces, ${ }^{14,15}$ in which $\gamma$ is equated to the integral of the excess interfacial stress. At present, there are two primary methods for the calculation of $\gamma_{s l}$ via molecular simulation. In the capillary fluctuation method (CFM) ${ }^{16}$ the interfacial stiffness is obtained from the spectrum of interfacial fluctuations. ${ }^{16}$ By determining the stiffness for a variety of interfacial orientations, accurate values of $\gamma_{s l}$ can be ob-

\footnotetext{
a) Author to whom correspondence should be addressed. Electronic mail: blaird@ku.edu.
}

tained. In the cleaving method, ${ }^{17,18} \gamma_{s l}$ is obtained using thermodynamic integration-directly calculating the reversible work per unit area required to continuously transform separate bulk crystal and melt systems into a single system containing an interface. Both of these methods, which have been applied to a number of mode ${ }^{18-23}$ and realistic ${ }^{16,24-27}$ potentials, are computationally intensive-the fluctuation method because of the large system sizes and long runs required to get adequate statistics, and the cleaving method because of the large number of simulations involved in the thermodynamic integration. Because of this difficulty, evaluations of $\gamma_{s l}$ for a given system, with the exception of the LennardJones (LJ) system, ${ }^{19,28}$ are generally restricted to a single point on the phase coexistence curve. As a consequence, more efficient simulation methods are needed to make practical the determination of $\gamma_{s l}$ over the full range of thermodynamic coexistence space for a system of interest.

Recently, Frolov and Mishin ${ }^{29}$ have demonstrated that $\gamma_{s l}$ for the solid-liquid interfaces of binary mixtures can be determined by direct integration along the temperaturecomposition curve at fixed pressure given a value of $\gamma_{s l}$ for one of the pure components. In this work, they determine $\gamma_{s l}$ for the (110) interface of $\mathrm{Cu} / \mathrm{Ag}$ mixtures of varying composition starting from a previously determined value ${ }^{30}$ of $\gamma_{s l}$ for pure $\mathrm{Cu}$ (110). Their approach, which we refer to as "GibbsCahn integration," is based upon Cahn's extension ${ }^{31}$ of the interfacial thermodynamics of Gibbs. ${ }^{1}$ Using the GibbsCahn formalism, differential changes in $\gamma_{s l}$ can be related to interfacial excess quantities that are readily calculated within an atomistic simulation, allowing for the determination of $\gamma_{s l}$ 
along the coexistence curve by direct integration. As such, this technique is analogous to the use of the Clapeyron equation to determine phase coexistence curves by direct integration along the curve itself. ${ }^{32,33}$ Frolov and Mishin have also employed Gibbs-Cahn integration to determine the surface free energy of $\mathrm{Cu}(110)$ as a function of temperature. In this work, we use the Gibbs-Cahn approach to demonstrate that $\gamma_{s l}$ can be obtained at a variety of temperatures along a temperature-pressure coexistence curve by integration of the excess interfacial energy and stress along that curve, provided that the value of $\gamma_{s l}$ has been previously determined for one temperature (for example, by CFM or cleaving). The result is a determination of $\gamma_{s l}$ as a function of coexistence temperature with a computational effort that is not significantly more than the calculation of a single $\gamma_{s l}$ value by CFM or cleaving.

The rest of the paper is organized as follows: in Sec. I, we describe in some detail the Gibbs-Cahn formalism and in Sec. II, we apply this formalism to the LJ system studied previously by Broughton and Gilmer, ${ }^{17}$ Davidchack and Laird, ${ }^{19}$ and Morris and Song. ${ }^{21}$ Starting with the values of $\gamma_{s l}$ at the triple-point temperature for the (100), (110), and (111) orientations determined in Ref. 19 using the cleaving method (and confirmed by CFM in Ref. 21), we calculate the excess interfacial energy and stress for several points along the coexistence curve and use this data to determine the value of $\gamma_{s l}$ at these points by Gibbs-Cahn integration. The values are in good agreement with those calculated by cleaving at temperatures above the triple point, but were obtained with significantly reduced computational effort. In Sec. III we conclude.

\section{GIBBS-CAHN FORMALISM}

In his formulation of the thermodynamics of planar interfaces, Gibbs projects all of the interfacial properties of a system onto a mathematical surface (called the Gibbs dividing surface) separating the two phases (here, solid and liquid). Using this dividing surface one can define interfacial excess quantities, such as the interfacial excess energy, $e$, entropy, $\eta$, and particle numbers, $\Gamma^{k}$

$$
\begin{aligned}
& e=\left(E-\rho_{s}^{E} V_{s}-\rho_{l}^{E} V_{l}\right) / A, \\
& \eta=\left(S-\rho_{s}^{S} V_{s}-\rho_{l}^{S} V_{l}\right) / A, \\
& \Gamma^{k}=\left(N^{k}-\rho_{s}^{k} V_{s}-\rho_{l}^{k} V_{l}\right) / A,
\end{aligned}
$$

where $A$ is the interfacial area, $N^{k}$ is the number of particles of type $k$, and $\rho^{E}, \rho^{S}$, and $\rho^{k}$ are the bulk densities of the energy, entropy, and number of particles of type $k$, respectively. The subscripts $l$ and $s$ refer, respectively, to the bulk liquid and solid. The volumes of the bulk phases, $V_{l}$ and $V_{s}$ are determined by the position of the Gibbs dividing surface. In all of these cases, the interfacial excess of a given quantity is defined as the difference between the actual value of that quantity and its value obtained by assuming bulk values up to the dividing surface. Note that, by construction the excess interfacial volume is zero within the Gibbs formalism. For a single component system, the interfacial free energy at a temperature $T$ is given by

$$
\gamma=e-T \eta-\mu \Gamma,
$$

where $\mu$ is the chemical potential of the substance. In this equation $\gamma$ is independent of the choice of dividing surface, but $e, \eta$, and $\Gamma$ are not. Typically, the Gibbs dividing surface for a pure system is chosen such that the excess number of particles $(\Gamma)$ vanishes, yielding

$$
\gamma=e-T \eta
$$

Note that, in this work we are focusing on systems in which the bulk phases are under hydrostatic stress. For details of the formalism for systems in which one or both coexisting phases are solids under nonhydrostatic stress, see Refs. 29 and 30.

In a seminal paper, ${ }^{31}$ Cahn reformulated and generalized Gibb's interface thermodynamics by eliminating the artificial construction of the dividing surface. For an $r$-component system containing a planar interface, the total Gibbs energy is given by

$$
G=E-T S+P V,
$$

where $P, T, E, S$, and $V$ are the pressure, temperature, internal energy, entropy, and volume, respectively. Without the interface the Gibbs energy would be equal to that of the bulk phases

$$
G_{b}=\sum_{k=1}^{r} \mu^{k} N^{k}
$$

where $\mu^{k}$ is the chemical potential of particles of type $k$. Assuming that the solid phase is under hydrostatic stress, the interfacial free energy $\gamma$ is given by the difference (per unit area) between the Gibbs energy of the system with the interface and that of the bulk

$$
\gamma A=G-G_{b}=E-T S+P V-\sum_{k=1}^{r} \mu^{k} N^{k} .
$$

The differential of this quantity is

$$
\begin{aligned}
d(\gamma A)= & d E-T d S-S d T+P d V+V d P-\sum_{i=1}^{r} \mu^{k} d N^{k} \\
& -\sum_{k=1}^{r} N^{k} d \mu^{k} .
\end{aligned}
$$

For a system containing a planar interface in which at least one of the coexisting phases is a crystal, the differential for the energy, under hydrostatic conditions in the solid, is given by $^{30}$

$$
d E=T d S-P d V+\sum_{i, j=1,2}\left(\sigma_{i j}+\delta_{i j} P\right) V d \epsilon_{i j}+\sum_{k=1}^{r} \mu^{k} d N^{k}
$$

where $\sigma_{i j}$ and $\epsilon_{i j}$ are the $i j$ components of the stress and strain tensors, respectively, and $i$ and $j$ are elements of the set $\{1,2\}$, which represent two orthogonal directions perpendicu- 
lar to the interface normal. Substituting Eq. (10) into Eq. (9) gives

$$
\begin{aligned}
d(\gamma A)= & -S d T+V d P+\sum_{i, j=1,2}\left[\left(\sigma_{i j}+\delta_{i j} P\right) V\right] d \epsilon_{i j} \\
& -\sum_{k=1}^{r} N^{k} d \mu^{k} .
\end{aligned}
$$

For a solid-liquid interface, in addition to Eq. (11), we have two additional equations, namely, the Gibbs-Duhem equations for the hydrostatic bulk solid and bulk liquid

$$
0=-S_{s} d T+V_{s} d P-\sum_{k=1}^{r} N_{s}^{k} d \mu^{k}
$$

and

$$
0=-S_{l} d T+V_{l} d P-\sum_{k=1}^{r} N_{l}^{k} d \mu^{k} .
$$

Here $s$ and $l$ indicate bulk solid and liquid quantities, respectively.

Equations (11)-(13) form a set of three simultaneous linear equations. Using Cramer's rule, Cahn showed that we can eliminate any pair of differentials $d x$ and $d y$ (e.g., $d P$ and $\left.d N^{k}\right)$ to give

$$
\begin{aligned}
d(\gamma A)= & -[S / X Y] d T+[V / X Y] d P \\
& +\sum_{i, j=1,2}\left[\left(\sigma_{i j}+\delta_{i j} P\right) V / X Y\right] d \epsilon_{i j}-\sum_{k=1}^{r}\left[N^{k} / X Y\right] d \mu^{k},
\end{aligned}
$$

where $X$ and $Y$ are the variables conjugate to the displacements $d x$ and $d y$, and the notation $[A / X Y]$ is defined as

$$
[A / X Y]=\frac{\left|\begin{array}{ccc}
A & X & Y \\
A_{l} & X_{l} & Y_{l} \\
A_{s} & X_{s} & Y_{s}
\end{array}\right|}{\left|\begin{array}{ll}
X_{l} & Y_{l} \\
X_{s} & Y_{s}
\end{array}\right|},
$$

where, again, $s$ and $l$ denote bulk solid and bulk liquid and quantities without these designations refer to the whole system (solid+liquid+interface). For a single component system $(r=1)$, a convenient choice is $X=N$ and $Y=V$. This choice is equivalent to choosing a Gibbs dividing surface in which the excess number of particles is zero $(\Gamma=0)$. With this choice the $d P$ and $d \mu$ terms in Eq. (14) are identically zero (because the determinant in Eq. (15) is zero if two columns are identical), giving

$$
d(\gamma A)=-[S / N V] d T+\sum_{i, j=1,2}\left[\left(\sigma_{i j}+\delta_{i j} P\right) V / N V\right] d \epsilon_{i j} .
$$

The determinant $[S / N V]$ reduces to the total excess entropy $A \eta$, as defined in Eq. (3). Because we assume that the system is hydrostatic, the second term on the right-hand side of Eq. (16) can be obtained as follows:

$$
\begin{aligned}
{\left[\left(\sigma_{i j}+\delta_{i j} P\right) V / N V\right] } & =\frac{\left|\begin{array}{ccc}
\left(\sigma_{i j}+\delta_{i j} P\right) V & N & V \\
0 & N_{s} & V_{s} \\
0 & N_{l} & V_{l}
\end{array}\right|}{\left|\begin{array}{ll}
N_{s} & V_{s} \\
N_{l} & V_{l}
\end{array}\right|} \\
& =\left(\sigma_{i j}+\delta_{i j} P\right) V .
\end{aligned}
$$

For the high-symmetry interface orientations considered in this work, $\sigma_{12}=\sigma_{21}=0$. Further, mechanical equilibrium at the interface guarantees that $\sigma_{33}=-P_{z z}=-P$ at the interface, so we have

$$
d(\gamma A)=-A \eta d T+\left(\sigma_{11}+P\right) V d \epsilon_{11}+\left(\sigma_{22}+P\right) V d \epsilon_{22} .
$$

The strain can be related to the change in the interfacial area as the crystal expands as one moves along the coexistence curve

$$
d \epsilon_{11}=d \epsilon_{22}=\frac{d A}{2 A} .
$$

Substituting this into Eq. (18) and dividing by $A$ gives

$$
\begin{aligned}
\frac{1}{A} d(\gamma A) & =-\eta d T+\frac{1}{A}\left(\sigma_{11}+\sigma_{22}+2 P\right) V \frac{d A}{2 A} \\
& =-\eta d T+\tau \frac{d A}{A},
\end{aligned}
$$

where the excess interfacial stress $\tau$ is defined in the usual way

$$
\tau=\frac{1}{A}\left(\frac{\sigma_{11}+\sigma_{22}}{2}+P\right) V=\int_{-\infty}^{\infty}\left[\left(P_{z z}-\frac{P_{x x}+P_{y y}}{2}\right)\right] d z,
$$

where $P_{z z}$ and $\left(P_{x x}+P_{y y}\right) / 2$ are the pressure components normal and transverse to the interface, respectively.

Use of Eq. (20) requires knowledge of the excess interfacial entropy $\eta$, which is not readily obtainable from simulation. For the current purposes, there are two ways to remedy this problem. One is to replace $\eta$ in Eq. (20) with $(e-\gamma) / T$ [Eq. (5)]. This transforms Eq. (20) into a more complicated differential equation. To remedy this, Frolov and Mishin, in a paper on surface free energy, ${ }^{30}$ and Baidakov et $a l$, in the context of liquid-vapor interfaces ${ }^{34}$ combine Eq. (5) $(\gamma=e-T \eta)$ with the fact that $\eta=-(d \gamma / d T)_{A}$ [from Eq. (20)] to derive

$$
\frac{1}{A} d(\gamma A / T)=-\frac{e}{T^{2}} d T+\frac{\tau}{T} \frac{d A}{A},
$$

which relates changes in $\gamma$ to the more easily obtainable excess interfacial energy, $e$. The derivation of this equation is analogous to the derivation of the Gibbs-Helmholtz equation in thermodynamics. ${ }^{35}$

After dividing both sides of Eq. (22) by $d T$, taken along the coexistence curve, and recognizing that, for the cubic 
crystals considered here, the interfacial area $A$ is proportional to $\rho_{s}^{-2 / 3}$, where $\rho_{s}$ is the number density of the solid, we obtain

$$
\left[\frac{d\left(\gamma_{g} / T\right)}{d T}\right]_{\text {coex }}=-\rho_{s}^{-2 / 3}\left[\frac{e}{T^{2}}+\frac{2 \tau}{3 \rho_{s} T}\left(\frac{d \rho_{s}}{d T}\right)_{\text {coex }}\right],
$$

where $\gamma_{g}$ is the usual interfacial free energy per surface atom defined by Turnbull: ${ }^{36} \gamma_{g}=\rho_{s}^{-2 / 3} \gamma$. Equation (23) is the central equation for this study. If we know the value of $\gamma$ at any point on the coexistence curve, we can integrate Eq. (23) to find $\gamma$ at any other point of the coexistence curve provided we have data for the excess interfacial energy $(e)$ and stress $(\tau)$ along the curve. Note that for a system in which the bulk solid is under hydrostatic stress, the value of the $\tau$ is independent of the definition of the Gibbs dividing surface (or, in the Cahn formalism, on the specific choice of $X$ and $Y$ ); however, the quantities $e$ and $\eta$ are not. Therefore, for Eqs. (20), (22), and (23) to be valid, $e$ and $\eta$ must be calculated using a dividing surface defined by $\Gamma=0[$ or $(X, Y)=(N, V)]$.

Recent work has demonstrated a strong linear correlation between Turbull's interfacial free energy per surface atom $\gamma_{g}$ and the melting point of a material. ${ }^{37,38}$ This correlation can be understood by examining the form of Eq. (23). A linear correlation of $\gamma_{g}$ with $T$ would be exact if the right-hand side of Eq. (23). were zero, which is identically the case for systems with inverse-power potentials $u(r)=\epsilon(\sigma / r)^{n}$, which includes the hard-sphere system in the limit that $n \rightarrow \infty .{ }^{20}$ Thus, the magnitude of the right-hand side of Eq. (23) gives an indication of the deviation from the relation $\gamma_{g} \propto T$.

\section{RESULTS FOR THE LJ SYSTEM}

For our study, we use a LJ pair potential due to Broughton and Gilmer ${ }^{39}$

$$
u(r)=\left\{\begin{array}{l}
4 \epsilon\left[\left(\frac{\sigma}{r}\right)^{12}-\left(\frac{\sigma}{r}\right)^{6}\right]+C_{1}, \quad r \leq 2.3 \sigma \\
C_{2}\left(\frac{\sigma}{r}\right)^{12}+C_{3}\left(\frac{\sigma}{r}\right)^{6} \\
+C_{4}\left(\frac{r}{\sigma}\right)^{2}+C_{5}, \quad 2.3 \sigma<r<2.5 \sigma \\
0, \quad 2.5 \sigma \leq r,
\end{array}\right.
$$

where $\quad C_{1}=0.016132 \epsilon, \quad C_{2}=3136.6 \epsilon, \quad C_{3}=-68.069 \epsilon$, $C_{4}=-0.083312 \epsilon$, and $C_{5}=0.74689 \epsilon$. (Note: the sign of $C_{4}$ was incorrectly reported as positive in Broughton and Gilmer's original publication.) This potential is constructed so that both the potential and the force smoothly go to zero at $r=2.5 \sigma$.

This potential has an advantage for the present study because its interfacial thermodynamics have been previously well characterized. In 1986, this system was employed by Broughton and Gilmer $^{17}$ in the first direct calculation of $\gamma_{s l}$ by computer simulation, in which they introduced the concept of cleaving potentials. The values of the reduced solidliquid interfacial free energy $\gamma_{s l}^{*}=\gamma \sigma^{2} / \epsilon$ were determined at the triple point $\left(T^{*}=k T / \epsilon=0.617 ; \rho^{*}=\rho \sigma^{3}=0.945\right)$ to be 0.34(2), 0.36(2), and 0.35(2) for the (100), (110), and (111) interfaces, respectively. The numbers in parentheses give the uncertainties in the last digits shown. As can be seen, the precision of their method was not sufficient to definitively resolve the anisotropy in $\gamma_{s l}$. Subsequently, in 2003 Davidchack and Laird ${ }^{19}$ used their cleaving wall method, developed earlier for the hard-sphere system, ${ }^{18}$ to determine $\gamma_{s l}$ for this $\mathrm{LJ}$ system at $T^{*}=0.617,1.00$, and 1.50 along the coexistence curve. This cleaving wall method is an extension of the cleaving potentials used by Broughton and Gilmer. At $T^{*}=0.617$, the $\gamma_{s l}^{*}$ values were determined to be $0.371(3)$, $0.360(2)$, and $0.347(3)$ for the (100), (110), and (111) interfaces, respectively. These values are consistent with the earlier estimates of Broughton and Gilmer, but are nearly an order of magnitude more precise, allowing for resolution of the orientational anisotropy. (Note that, Davidchack and Laird $^{19}$ found that, at $T^{*}=0.617$, the coexistence pressure is negative (though small) and that a better estimate of the triple point temperature is slightly higher at $T^{*}=0.618$. However, the change in $\gamma_{s l}$ due to this temperature difference is much smaller than the statistical error in these quantities.) Recently, Morris and Song ${ }^{21}$ examined this system at the triple point temperature using the CFM, obtaining $\gamma_{s l}$ $=0.369(8), 0.361(8)$, and $0.355(8)$ for the (100), (110), and (111) interfaces, respectively. These CFM values agree within the error bars with those of Davidchack and Laird using cleaving walls.

Starting from the values determined by Davidchack and Laird for $\gamma_{s l}$ at the triple point, we can use Eq. (23) to determine the value of $\gamma_{s l}$ at higher temperatures along the coexistence curve. To do this we have calculated the crystal density, $\rho_{c}$ and the excess interfacial energy $e$ and stress $\tau$ at several temperatures $\left(T^{*}=0.618,0.809,1.0,1.25\right.$, and 1.5) along the coexistence curve. These calculations are performed using NVT molecular-dynamics simulation with a Nosé-Hoover thermostat ${ }^{40}$ with system sizes of approximately $N=120000$ particles-this number varies slightly from interface to interface. Such large systems were used to minimize the dependence of the coexistence conditions on interface orientation and to facilitate precise calculation of the coexistence conditions. Additional simulation details including the determination of the coexistence densities (summarized in Table I), as well as the creation of the equilibrium interfaces, can be found in Refs. 12 and 41.

The excess interfacial energies were computed in the following manner. The bulk densities of the solid and liquid phases ( $\rho_{s}$ and $\rho_{l}$, respectively), as well as the respective bulk potential energy densities $\left(\rho_{s}^{E}\right.$ and $\left.\rho_{l}^{E}\right)$ were determined by constructing filtered interfacial profiles of potential energy and density as functions of distance along the interface normal (here taken to be $z$ ). The details as to our protocol for the construction of profiles using finite impulse response filters are described in Refs. 12 and 41. As an example, the interfacial energy profiles for the (100), (110), and (111) interfacial orientations at the triple point temperature $\left(T^{*}=0.618\right)$ are plotted in Fig. 1. (The energy profiles for the other temperatures examined in this study have been deposited as supplementary material. ${ }^{42}$ ) These profiles are determined by averaging over $10^{4}$ times steps. (One time step is 0.01 $\sqrt{m \sigma^{2} / \epsilon}$, where $m$ is the mass of the particles.) To calculate the bulk densities and energies, we average these profiles 
TABLE I. Coexistence densities, excess interfacial energy and excess interfacial stress for the truncated LJ system as a function of temperature. Numbers in parentheses are the estimated statistical errors $(95 \%$ confidence level) in the last digit shown.

\begin{tabular}{|c|c|c|c|c|c|c|c|c|}
\hline \multirow[b]{2}{*}{$k T / \epsilon$} & \multirow[b]{2}{*}{$\rho_{s}^{*}=\rho_{s} \sigma^{3}$} & \multirow[b]{2}{*}{$\rho_{f}^{*}=\rho_{f} \sigma^{3}$} & \multicolumn{3}{|c|}{ Excess interface energy: $e^{*}=e \sigma^{2} / \epsilon$} & \multicolumn{3}{|c|}{ Excess interface stress: $\tau^{*}=\tau \sigma^{3} / \epsilon$} \\
\hline & & & $(100)$ & (110) & (111) & $(100)$ & (110) & (111) \\
\hline 0.618 & $0.9445(1)$ & $0.8282(3)$ & $0.134(4)$ & $0.188(3)$ & $0.186(3)$ & $-0.182(10)$ & $-0.80(2)$ & $-0.86(2)$ \\
\hline 0.809 & $0.9744(1)$ & $0.8829(2)$ & $0.081(2)$ & $0.157(3)$ & $0.162(3)$ & $-0.056(10)$ & $-0.81(2)$ & $-0.88(2)$ \\
\hline 1.00 & $1.0044(1)$ & $0.9288(3)$ & $0.060(3)$ & $0.153(4)$ & $0.157(4)$ & $0.008(17)$ & $-0.85(2)$ & $-0.93(3)$ \\
\hline 1.25 & $1.0411(1)$ & $0.9644(3)$ & $0.036(3)$ & $0.162(5)$ & $0.164(5)$ & $0.155(17)$ & $-0.96(3)$ & $-1.02(4)$ \\
\hline 1.5 & $1.0743(1)$ & $1.0030(2)$ & $0.020(3)$ & $0.169(4)$ & $0.180(3)$ & $0.204(17)$ & $-1.01(3)$ & $-1.12(3)$ \\
\hline
\end{tabular}

over all regions outside of a $7 \sigma$ range of the interface location. Once the bulk densities and energies are determined, the excess interfacial energy is obtained using the equation

$$
e=\frac{1}{2 A}\left[E_{\text {total }}-\rho_{s}^{E} A L_{s}-\rho_{l}^{E} A\left(L-L_{s}\right)\right],
$$

where $E_{\text {total }}$ is the total average energy of the interfacial system, $L$ is the total length of the system normal to the interface, and $L_{s}$ is the length of the solid phase in the direction normal to the interface, which is determined by solving the equation for the Gibbs dividing surface

$$
0=N-A\left[L_{s} \rho_{s}-\left(L-L_{s}\right) \rho_{l}\right] .
$$

The factor of 2 in the right-hand side of Eq. (24) is due to the fact that the simulation box contains two interfaces.

The values of the interfacial excess stress were determined by first constructing filtered interfacial stress profiles [defined as the integrand in Eq. (21)], which are then integrated over a region completely containing the interface. We have determined that the region defined by $\pm 7 \sigma$ from the dividing surface was sufficient to guarantee convergence of interfacial quantities. Because the stress is zero in both the bulk solid and the bulk liquid, the excess interfacial stress is independent of the dividing surface. The stress profiles for the (100), (110), and (111) interfaces at $T^{*}=0.618$ are shown in Fig. 2. (The stress profiles for the other temperatures examined in this study have been deposited as supplementary

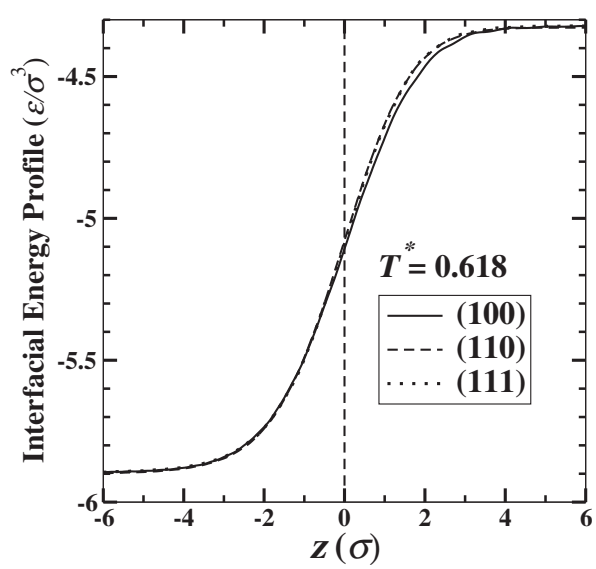

FIG. 1. Interfacial energy profiles for the LJ solid-liquid interface at the triple point $\left(T^{*}=0.618\right)$ for the (100), (110), and (111) interfacial orientations. The interface normal direction is denoted by $z$. The vertical dashed line at $z=0$ indicates the position of the Gibbs dividing surface. material. ${ }^{42}$ ) The values for the excess interfacial energy and stress (in reduced units) for all orientations and temperatures studied are tabulated in Table I and plotted as functions of temperature in Figs. 3 and 4, respectively.

In examining Figs. 3 and 4 some interesting trends emerge. First, the excess interfacial energies and stresses for the (100) orientations are significantly different than those for the (110) and (111) orientations, which have similar values. In the case of the excess energies (Fig. 3), the values for the (110) and (111) interfaces are nearly independent of temperature, whereas the values for the (100) interface decrease significantly as temperature is raised. For the stresses (Fig. 4), the values for both the (110) and (111) interfaces are negative and decrease with increasing temperature, whereas those for the (100) interface start out negative at the triple point, but increase with temperature and become positive at about $T^{*}=1.0$.

To integrate Eq. (23) to obtain $\gamma_{g} / T$ and subsequently $\gamma_{s l}$, it is necessary to first find $d \rho_{s} / d T$ along the coexistence line. This is done by fitting the data for $\rho_{s}^{*}$ in Table I to a quadratic function in $T^{*}$ using least-squares regression. This gives

$$
\left(\frac{d \rho_{s}^{*}}{d T^{*}}\right)_{\text {coex }}=0.1875-0.37730 T^{*} .
$$

Because of the quality of this fit and the extremely small statistical error present in $\rho_{s}^{*}$, the estimated errors in $d \rho_{s}^{*} / d T^{*}$ are small enough that we can ignore them in subsequent error propagation.

Figure 5 shows the derivative $d\left(\gamma_{g}^{*} / T^{*}\right) / d T^{*}$ along the coexistence line [Eq. (23)] as a function of temperature. Note that this quantity decreases significantly to zero as the temperature is raised. This reflects the fact that, as $T$ increases away from the triple point, the interfacial thermodynamics of the LJ system should eventually approach that of a system with an inverse-12th power repulsion, for which $\gamma_{g}^{*} / T^{*}$ is a constant. $^{20}$ [That is, Eq. (23) is identically zero for an inverse-power potential.] Also shown in Fig. 5 is the contribution to this derivative due to the excess interfacial energy [the first term on the right-hand side of Eq. (23)]. For the (100) interface, the derivative $d\left(\gamma_{g}^{*} / T^{*}\right) / d T^{*}$ [Eq. (23)] is almost completely dominated by the excess energy contribution, whereas, for the (110) and (111) interfaces, the excess energy contribution to Eq. (23) is more negative than the total derivative itself. This implies that, in the (110) and (111) interfaces, the excess stress contribution to the deriva- 


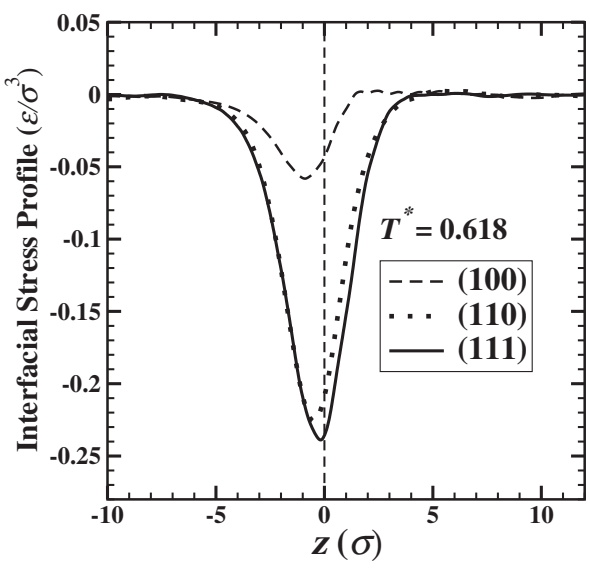

FIG. 2. Interfacial stress profiles for the LJ solid-liquid interface at the triple point $\left(T^{*}=0.618\right)$ for the (100), (110), and (111) interfacial orientations. The interface normal direction is denoted by $z$. The vertical dashed line at $z=0$ indicates the position of the Gibbs dividing surface.

tive is opposite in sign to that of the excess energy and, at high temperatures, approaches the excess energy contribution in magnitude.

It is interesting to note from Fig. 5 that the anisotropy in the quantity $d\left(\gamma_{g}^{*} / T^{*}\right) / d T^{*}$ is relatively small, especially at the higher temperatures. This is in stark contrast to the large anisotropies seen in the excess interfacial energies and stresses (see Figs. 1 and 2) that combine to determine $d\left(\gamma_{g}^{*} / T^{*}\right) / d T^{*}$ in Eq. (23). For a system in which the solid is under hydrostatic stress, the excess interfacial stress, $\tau$, is independent of the choice of dividing surface (or $X$ and $Y$ in the Cahn formalism), which implies that the second term on the right-hand side of Eq. (23) is also independent of this choice. The excess interfacial energy, however, is not independent of the dividing surface. The fact that $d\left(\gamma_{g}^{*} / T^{*}\right) / d T^{*}$ must also be independent of dividing surface may seem inconsistent with the fact that the first and second terms on the right-hand side of Eq. (23) are, respectively, dependent and independent of the choice of the dividing surface (implying that the sum is dependent on dividing surface) for a hydrostatically stressed solid. However, it must be noted that a change in the dividing surface will also change the specific

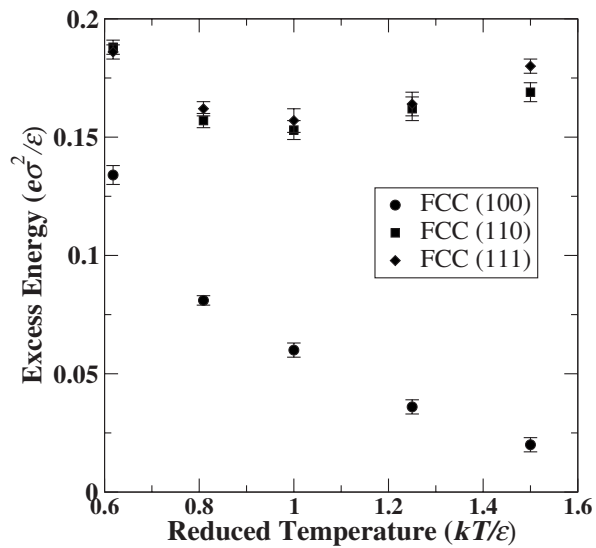

FIG. 3. Interfacial excess energy (in reduced units) for the LJ solid-liquid interface as a function of reduced temperature $\left(T^{*}=k T / \epsilon\right)$ for the (100), (110), and (111) interfacial orientations. The error bars represent $2 \sigma$ deviations.

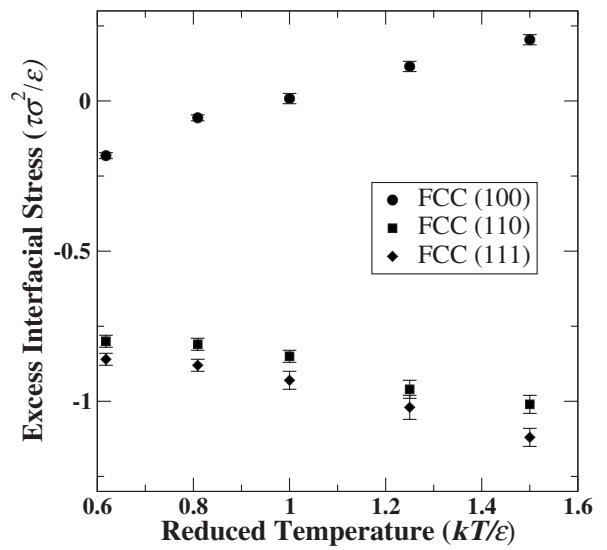

FIG. 4. Interfacial excess stress (in reduced units) for the LJ solid-liquid interface as a function of reduced temperature $\left(T^{*}=k T / \epsilon\right)$ for the (100), (110), and (111) interfacial orientations. The error bars represent $2 \sigma$ deviations.

form of Eq. (23), so that the first term would be some combination of the excess interfacial energy and excess particle number. It is this combination that is invariant to changes in the dividing surface, not the specific value of the excess interfacial energy. However, the question as to why these two terms, which are intrinsically anisotropic, should combine to give a relatively isotropic sum is, at present, open.

The values of $\gamma_{s l}$ above the triple point $\left(T^{*}=0.618\right)$ are obtained by numerically integrating the data in Fig. 5 to directly obtain $\gamma_{g} / T$, from which $\gamma_{s l}$ is easily determined. The data points at $T^{*}=0.809$ and 1.25 were chosen because they lie halfway between the temperatures $\left(T^{*}=0.618,1.00\right.$, and 1.5) for which $\gamma_{s l}$ was calculated by cleaving. ${ }^{19}$ This allows for the use of Simpson's rule to integrate Eq. (23) to obtain $\gamma_{s l}$ at $T^{*}=1.0$ and 1.5. The Trapezoid Rule was used to obtain $\gamma_{s l}$ at the intermediate temperatures. The results of these integrations are summarized in Table II along with the values determined by cleaving in Ref. 19, for comparison. The integration errors for the Simpson's Rule calculation have been estimated to be approximately an order of magnitude smaller than the statistical errors in the calculated $\gamma_{s l}$ values. The Trapezoid Rule integration errors for the intermediate points are somewhat higher and are approximately the same size as

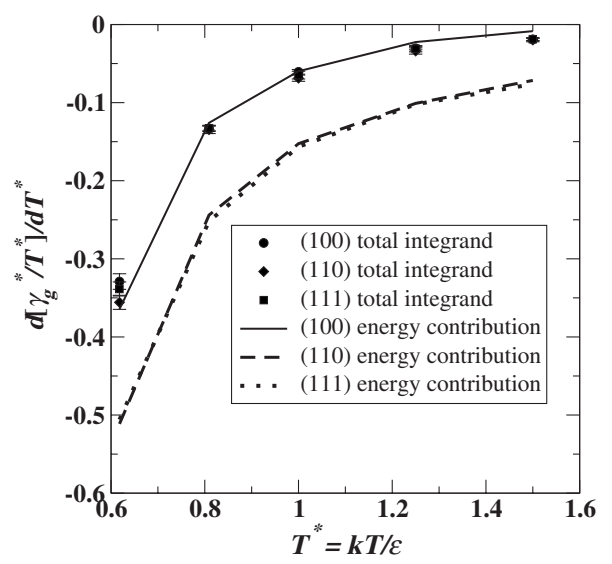

FIG. 5. The quantity $d\left(\gamma_{g}^{*} / T^{*}\right) / d T^{*}$ from Eq. (23) as a function of reduced temperature $\left(T^{*}=k T / \epsilon\right)$ for the (100), (110), and (111) interfacial orientations. The error bars represent $2 \sigma$ deviations. 
TABLE II. Interfacial free energy calculated both by Gibbs-Cahn integration (using the trapezoid rule) and by cleaving, as well as the excess interfacial entropy, for the truncated LJ system at temperatures along the solidliquid coexistence line. Numbers in parentheses are the estimated errors (95\% confidence level) in the last digit shown.

\begin{tabular}{lcccc}
\hline \hline \multirow{2}{*}{$k T / \epsilon$} & Orientation & $\gamma^{*}$ (this work) & $\gamma^{* \mathrm{a}}$ & $\eta^{*}$ \\
\hline \multirow{2}{*}{0.618} & $(100)$ & $\ldots$ & $0.371(3)$ & $-0.383(8)$ \\
& $(110)$ & $\ldots$ & $0.360(3)$ & $-0.278(7)$ \\
& $(111)$ & $\ldots$ & $0.347(3)$ & $-0.260(7)$ \\
0.809 & $(100)$ & $0.462(4)$ & $\ldots$ & $-0.470(6)$ \\
& $(110)$ & $0.444(4)$ & $\ldots$ & $-0.355(6)$ \\
1.00 & $(111)$ & $0.428(4)$ & $\ldots$ & $-0.328(6)$ \\
& $(100)$ & $0.570(5)$ & $0.562(6)$ & $-0.510(6)$ \\
& $(110)$ & $0.545(5)$ & $0.543(6)$ & $-0.392(6)$ \\
1.25 & $(111)$ & $0.525(5)$ & $0.508(8)$ & $-0.368(6)$ \\
& $(100)$ & $0.716(7)$ & $\ldots$ & $-0.544(5)$ \\
& $(110)$ & $0.682(7)$ & $\ldots$ & $-0.416(7)$ \\
1.5 & $(111)$ & $0.657(7)$ & $\ldots$ & $-0.394(7)$ \\
& $(100)$ & $0.868(8)$ & $0.84(2)$ & $-0.565(6)$ \\
\hline \hline \multirow{2}{*}{1.5} & $(110)$ & $0.826(8)$ & $0.82(2)$ & $-0.438(6)$ \\
& $(111)$ & $0.797(9)$ & $0.75(3)$ & $-0.411(6)$ \\
\hline
\end{tabular}

${ }^{\mathrm{a}}$ Reference 19.

the statistical errors. At $T^{*}=1.0$, the values from Gibbs-Cahn integration agree within the error bars with those from cleaving, except for the values for (111), which are slightly higher in the current work. At $T^{*}=1.5$, the values for (110) agree within the error bars for the two methods, but the current values for (100) and (111) are higher by 3\%-5\% than those for cleaving, although the error bars on the latter are quite large. The origin of these discrepancies is not clear, although the current method, in addition to being less computationally intensive than cleaving, appears also to have significantly smaller statistical error.

To get an estimate of the contributions that the excess energy and stress have on the temperature dependence of $\gamma_{s l}$, one can calculate the values of $\gamma_{s l}$ assuming that the righthand side of Eq. (23) is identically zero. Such a calculation overestimates $\gamma_{s l}$ by $10 \%-15 \%$ at the highest temperatures, depending upon the interface. This shows that assuming a linear dependence of $\gamma_{g}=\rho^{-2 / 3} \gamma_{s l}$ with temperature gives a rough estimate of the value of $\gamma_{s l}$, but that more accurate values require the more detailed contribution of excess interfacial energy and stress considered here.

Also shown in Table II, are the results for the interfacial excess entropy, $\eta$, for this choice of dividing surface. These excess entropies are calculated from the excess energies and free energies using Eq. (5). In all cases, $\eta$ is negative, which is consistent with the theory of interfacial free energy due to Spaepen, ${ }^{43}$ and decreases (becomes more negative) with increasing temperature. Like the excess interfacial energies and stresses, the excess entropies are much more anisotropic than $\gamma_{s l}$ itself.

\section{SUMMARY AND DISCUSSION}

In this work, we have demonstrated that the solid-liquid interfacial free energy $\gamma_{s l}$ for the LJ system can be accurately and efficiently calculated along the pressure-temperature coexistence curve via molecular simulation using Gibbs-Cahn

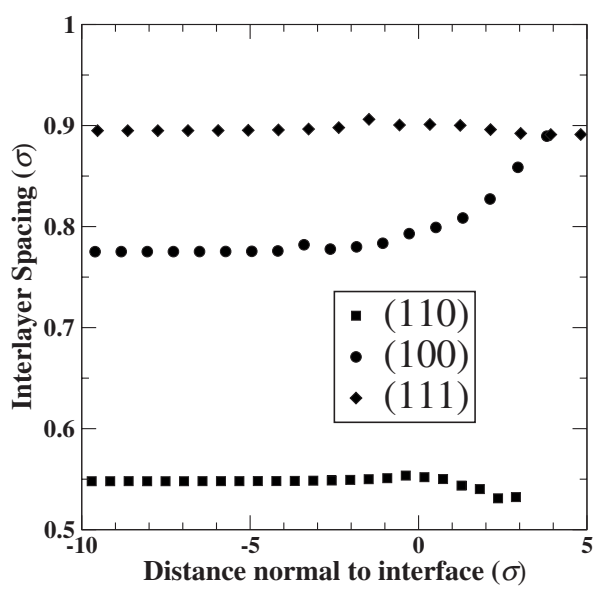

FIG. 6. The interplane spacing as a function of distance normal to the interface for the (100), (110), and (111) LJ solid-liquid interfaces.

integration. This technique requires determination of the excess interfacial energy and stress along the coexistence curve, as well as a previously determined value for $\gamma_{s l}$ at one reference temperature, which in our case is that of the $\mathrm{LJ}$ triple point $\left(T^{*}=0.618\right)$. Our calculated values for $\gamma_{s l}$ at $T^{*}$ $=1.0$ and 1.5 are in good agreement with those determined previously using the cleaving method, ${ }^{19}$ but were obtained with significantly less computational effort than required by either the cleaving method or the CFM. This work complements a recent calculation by Frolov and Mishin ${ }^{29}$ in which Gibbs-Cahn integration was used to determine $\gamma_{s l}$ along the temperature-composition coexistence curve for binary $\mathrm{Cu} / \mathrm{Ag}$ alloys.

One interesting observation from this work is that the excess interfacial stresses $(\tau)$, energies $(e)$, and entropies $(\eta)$ calculated here exhibit a far greater anisotropy than that seen in the interfacial free energy $\gamma_{s l}$ itself. Specifically, these quantities for the (100) interface are significantly different than those for the (110) and (111) interfaces, which are similar in magnitude and trend. These differences are both qualitative and quantitative. For example, the values of $\tau$ for the (110) and (111) interfaces are negative over the range of temperatures studied and decrease with increasing temperature. The excess stress for the (100) interface, on the other hand, increases with increasing temperature and is negative at the triple point $\left(T^{*}=0.618\right)$, but becomes positive above about $T^{*}=1.00$. One possible origin for this is the fact that, for fcc systems, the interplane spacing normal to the interface for the (100) interface orientation increases significantly as the interface is approached from the solid, whereas no significant increase is seen in either (111) or (110) — see Fig. 6. This increase in the interplanar spacing for a (100) fcc solid-liquid interface was also seen previously in simulations of hard spheres ${ }^{41}$ and aluminum. ${ }^{44}$ It is plausible to expect that such an expansion of the lattice near the interface should have a significant effect on the energetics and stress of the (100) interface relative to that for the other interfaces.

In this present study, the coexistence curve for this system was already known; however, in general, such information might not be a priori available. In such cases, if the interfacial free energy were known at one coexistence 
temperature/pressure, it would be possible to combine the Gibbs-Cahn integration scheme described here with GibbsDuhem integration $^{32,33}$ to simultaneously determine both the coexistence curve and the interfacial free energy along it. Also, the formalism is not restricted to solid-liquid interfaces and can be applied to solid-vapor, liquid-liquid, and liquidvapor interfaces without modification.

\section{ACKNOWLEDGMENTS}

M.A. acknowledges funding from the Department of Energy, Office of Basic Energy Sciences, under Contract No. DE-FG02-06ER46282. Y.Y. acknowledges support from the National Natural Science Foundation of China, Shanghai Project for the Basic Research (Contract No. 08JC1408400) and D. Y. Sun for helpful discussions. M.A. and B.B.L. acknowledge the U.S. Department of Energy (DOE)-sponsored Computational Materials Science Network (CMSN) program. The majority of the computations were carried out on the University of Leicester Mathematical Modeling Centre cluster (purchased through the HEFCE Science Research Investment Fund). Finally, B.B.L. gratefully acknowledges M.A. and the University of California, Davis for their hospitality during his Fall 2008 sabbatical.

${ }^{1}$ J. W. Gibbs, The Collected Works (Yale University Press, New Haven, CT, 1957), Vol. 1.

${ }^{2}$ K. F. Kelton, Solid State Phys. 45, 75 (1991).

${ }^{3}$ L. Gránásy and T. Pusztai, J. Chem. Phys. 117, 10121 (2002)

${ }^{4}$ S. Auer and D. Frenkel, Annu. Rev. Phys. Chem. 55, 333 (2004).

${ }^{5}$ A. Karma and W.-J. Rappel, Phys. Rev. E 57, 4323 (1998).

${ }^{6}$ W. J. Boettinger, S. R. Coriell, A. L. Greer, A. Karma, W. Kurz, M. Rappaz, and R. Trivedi, Acta Mater. 48, 43 (2000).

${ }^{7}$ H. Nam and D. J. Srolovitz, Phys Rev. B 76, 184114 (2007).

${ }^{8}$ A. W. Adamson and A. P. Gast, Physical Chemistry of Surfaces (WileyInterscience, New York, 1997).

${ }^{9}$ J. M. Howe, Interfaces in Materials (Wiley, New York, 1997).

${ }^{10}$ M. E. Glicksman and N. B. Singh, J. Cryst. Growth 98, 277 (1989).
${ }^{11}$ M. Muschol, D. Liu, and H. Z. Cummins, Phys. Rev. A 46, 1038 (1992).

${ }^{12}$ B. B. Laird and R. L. Davidchack, J. Phys. Chem. B 109, 17802 (2005).

${ }^{13} \mathrm{~W}$. A. Tiller, The Science of Crystallization: Microscopic Interfacial Phenomena (Cambridge University Press, New York, 1991).

${ }^{14}$ J. R. Henderson and F. van Swol, Mol. Phys. 51, 991 (1984).

${ }^{15}$ E. De Miguel and G. Jackson, Mol. Phys. 104, 3717 (2006).

${ }^{16}$ J. J. Hoyt, M. Asta, and A. Karma, Phys. Rev. Lett. 86, 5530 (2001).

${ }^{17}$ J. Q. Broughton and G. H. Gilmer, J. Chem. Phys. 84, 5759 (1986).

${ }^{18}$ R. L. Davidchack and B. B. Laird, Phys. Rev. Lett. 85, 4751 (2000).

${ }^{19}$ R. L. Davidchack and B. B. Laird, J. Chem. Phys. 118, 7651 (2003).

${ }^{20}$ R. L. Davidchack and B. B. Laird, Phys. Rev. Lett. 94, 086102 (2005).

${ }^{21}$ J. R. Morris and X. Song, J. Chem. Phys. 119, 3920 (2003).

${ }^{22}$ R. L. Davidchack, J. R. Morris, and B. B. Laird, J. Chem. Phys. 125, 094710 (2006).

${ }^{23}$ Y. Mu and X. Song, J. Chem. Phys. 124, 034712 (2006).

${ }^{24}$ J. J. Hoyt and M. Asta, Phys. Rev. B 65, 214106 (2002).

${ }^{25}$ M. Asta, J. J. Hoyt, and A. Karma, Phys. Rev. B 66, 100101(R) (2002).

${ }^{26}$ D. Y. Sun, M. Asta, and J. J. Hoyt, Phys. Rev. B 69, 174104 (2004).

${ }^{27}$ X. Feng and B. B. Laird, J. Chem. Phys. 124, 044707 (2006).

${ }^{28}$ C. A. Becker, D. Olmsted, M. Asta, J. J. Hoyt, and S. M. Foiles, Phys. Rev. Lett. 98, 125701 (2007).

${ }^{29}$ T. Frolov and Y. Mishin, J. Chem. Phys. 131, 054702 (2009).

${ }^{30}$ T. Frolov and Y. Mishin, Phys. Rev. B 79, 045430 (2009).

${ }^{31}$ J. W. Cahn, in Interfacial Segregation, edited by W. C. Johnson and J. M. Blakely (American Society for Metals, Metals Park, OH, 1979), pp. 3-23.

${ }^{32}$ D. A. Kofke, Mol. Phys. 78, 1331 (1993).

${ }^{33}$ D. A. Kofke, J. Chem. Phys. 98, 4149 (1993).

${ }^{34}$ V. G. Baidakov, S. P. Protsenko, and G. G. Chernykh, Russ. J. Phys. Chem. 80, 1519 (2006).

${ }^{35}$ R. A. Alberty and R. J. Silbey, Physical Chemistry, 3rd ed. (WileyInterscience, New York, 2000).

${ }^{36}$ D. Turnbull, J. Appl. Phys. 21, 1022 (1950).

${ }^{37}$ B. B. Laird, J. Chem. Phys. 115, 2887 (2001)

${ }^{38}$ R. M. Digilov, Physica B 352, 53 (2004).

${ }^{39}$ J. Q. Broughton and G. H. Gilmer, Acta Metall. 31, 845 (1983).

${ }^{40}$ W. G. Hoover, Phys. Rev. A 31, 1695 (1985).

${ }^{41}$ R. L. Davidchack and B. B. Laird, J. Chem. Phys. 108, 9452 (1998).

${ }^{42}$ See EPAPS supplementary material at http://dx.doi.org/10.1063/ 1.3231693 for plots of the excess interfacial energy and stress at all temperatures studied here.

${ }^{43}$ F. Spaepen, Acta Metall. 23, 729 (1975).

${ }^{44}$ J. D. Sturgeon, Ph.D. thesis, University of Kansas, 2002. 